\title{
Rich: Region-based Intelligent Cluster-Head Selection and Node Deployment Strategy in Concentric-based WSNs
}

\author{
Chung-Shuo FAN \\ Department of Computer Science, National Chiao Tung University, \\ EC 610, No. 1001, University Road, Hsinchu 30010, Taiwan, ROC \\ csfan@tp.edu.tw
}

\begin{abstract}
In a random deployment, sensor nodes are scattered randomly in the sensing field. Hence, the coverage can not be guaranteed. In contrast, the coverage of uniformly deployment is in general larger than the random deployment. However, uniformly deployment strategy may cause unbalanced traffic pattern in wireless sensor networks (WSNs). In this situation, larger load may be imposed to $\mathrm{CHs}$ (cluster heads) around the sink. Therefore, $\mathrm{CHs}$ close to the sink use up their energy earlier than those farther away from the sink. To overcome this problem, we propose a novel node deployment strategy in the concentric model, namely, Region-based Intelligent Cluster-Head selection and node deployment strategy (called Rich). The coverage, energy consumption and data routing issues are well investigated and taken into consideration in the proposed Rich scheme. The simulation results show that the proposed Rich alleviates the unbalanced traffic pattern significantly, prolongs network lifetime and achieves satisfactory coverage ratio.
\end{abstract}

Index Terms-Clustering methods; Energy consumption; Wireless sensor networks; Energy efficiency; Routing protocols.

\section{INTRODUCTION}

A wireless sensor network (WSN) is formed by a large number of connected sensor nodes that have the ability to sense, compute, and store sensed data as well as communicate with others via wireless communications. These characteristics allow WSNs to be used in a wide range of applications [1-4]. Node deployment [5-7] is one of the primary issues in WSNs. Appropriate node deployment strategies can reduce the overhead in terms of routing, data fusion, and prolong the network lifetime in WSNs.

WSNs can be classified into homogeneous or heterogeneous sensor networks. Even if several researches discuss heterogeneous WSNs, lots of current studies look into node deployment schemes in homogeneous WSNs because of less complexity and manageability. Hence, this paper investigates homogeneous sensor nodes in WSNs. These sensor nodes can be deployed in a random or deterministic manner. Although the random deployment method is preferable in many applications, the coverage can not be guaranteed. Therefore, deterministic deployment strategy should be investigated because a proper node deployment can increase the network lifetime in WSNs.

The crucial challenge in WSNs is energy efficiency because the individual nodes have limited battery. In a multi-hop WSN, the sensor nodes around the sink consume relatively more energy and use up their energy first. This many-to-one WSN may cause the 'energy hole problem'. It is due to the fact that these sensor nodes must forward the relay traffic from the rest of the sensor nodes. Hence, how to effectively balance the energy consumption in a WSN, and how to avoid the 'energy hole problem' become an important issue. To this end, this paper introduces a novel node deployment strategy in the concentric model called "Rich" (Region-based Intelligent Cluster-Head selection and node deployment strategy), which determines the node density in each region based on the energy consumption of $\mathrm{CHs}$, and aims at mitigating the 'energy hole problem'. More specifically, this paper provides explicit numerical calculations for the node density in each region to guarantee that all the sensor nodes exhaust their energy almost at the same time (i.e., the energy hole is relieved). The main contributions of this paper are listed as follows.

(1) Calculating the node density in each region based on the energy consumption of each $\mathrm{CH}$.

This result prevents the overloading of $\mathrm{CHs}$ around the sink, thereby alleviates the load of $\mathrm{CHs}$ near the sink and balances the energy consumption across the network.

(2) Finding out the area of region $_{x, j}$ is greater than the area of region $\boldsymbol{y}_{\mathbf{j}, j}$ if $\mathbf{x}>\mathbf{y}, \forall 1<x, y<k$.

Under the procedure of division (mention below in (1)), we demonstrate that the area of region $_{x, j}$ is greater than the area of region $_{y, j}$ if $\mathrm{x}>\mathrm{y}, \forall \mathrm{l}<x, y<k$, as shown in Theorem 1. This important finding also provides the guideline for deploying sensor nodes in the concentric-based WSN.

(3) Adopting multi-hop transmission in inter-region data routing.

To reduce the energy consumption of $\mathrm{CHs}$ in inter-region data routing, the proposed Rich utilizes multi-hop transmission. Therefore, the network lifetime of CHs away from the sink can prolong.

The rest of the paper is organized as follows. Section II surveys related works. Section III describes the network model and relevant assumptions. Section IV analyzes energy consumption of $\mathrm{CH}$ in each region. Based on the analysis results, the proposed Rich is explained in detail in Section V. Section VI shows the performance evaluations of the Rich and compares it to the EBDA scheme and Random scheme. Finally, the paper is concluded in Section VII.

\section{RELATED WORK}

A significant amount of research has studied the node deployment problem in terms of the network lifetime. The majority of the researches can be classified into the random deployment and the deterministic deployment [8].

Random deployment is more applicable in many scenarios where the area of interest (AOI) is hostile, or the 
sensing area is enormous. Liu [9] addressed the deployment issue to prolong the network lifetime in a multihop WSN. Simulation results show that the proposed algorithm have an energy-efficient clustering and gradient-based routing algorithm. Maleki and Pedram [10] determined the densities of sensor nodes at the beginning. They also provide a continuous space model in the random deployment that can be used to provide the minimum required energy depletion. Xin et al. [11] first studied the biased energy consumption rate (BECR) phenomenon in a multihop WSN. They consider the joint problem of relay node deployment and transmission power control in order to prolong the network lifetime. Kulkarni et al. [12] proposed a new deployment strategy, called Quasi Random Deployment (QRD), to improve the energy efficiency, increase the coverage, and extend the network lifetime. Random Deployment (RD) pattern in WSNs is analyzed as well. Tsai [13] investigated the sensing coverage for randomly distributed WSNs in the shadowed environments. The basic observation is to analyze the impact of the shadowing effects on the sensing coverage. Balister and Kumar [14] addressed the effects of placement errors and random failures on the density when sensors are deployed randomly versus deterministically.

Deterministic deployment can be applied in the conditions where the AOI is human accessible. Chen et al. [15] have studied the theoretical aspects of the non-uniform deployment scheme and addressed the energy hole problem in WSNs. Wang et al. [16] have provided theoretical formulations for the coverage and lifetime of a WSN based on 2D Gaussian distribution. Aderohunmu et al. [17] proposed a deterministic energy-efficient clustering model that better utilizes the energy in WSNs. This protocol is dynamic and self-organizing protocol, which is able to distribute the energy depletion uniformly among the sensor nodes. Ganesan et al. [18] investigated an optimization problem that considers jointly node placement, transmission structure in the data gathering WSN. They have simplified problem of optimal placement in the one-dimensional (1-D) case. They further extend the results to the 2-D case. Our previous work [19] presented a cluster-based routing protocol (called ACT) in the rectangle topology. The aim of the proposed ACT is to calculate the cluster radius in order to balance the energy dissipation of each $\mathrm{CH}$. Simulation results show that ACT can efficiently reduce the energy consumption of $\mathrm{CHs}$ around the sink while increasing the network lifetime. Our previous work inspired us to propose new cluster-based routing method in concentric-based WSNs. Senouci et al. [20] proposed an uncertainty-aware deployment algorithm (called EBDA), which determines the locations and minimum number of sensor nodes so as to achieve full coverage. Moreover, they have studied several issues, such as connectivity, preferential coverage, sensor reliability and challenging environments.

\section{PRELIMINARIES}

In this paper, we consider a concentric model of total radius $(R)$ with a static sink located at the center of circular sensing area. A concentric model is further considered by partitioning the circular sensing area into several levels. The $i$ th level is denoted as $L_{i}$ with each level having the same width $\left(r_{i}\right)$. A level $L_{i}$ is further divided into several region (region $\left._{i, j}\right), \forall j=4 i$, where $i$ donotes the $i$ th level and $j$ represents the order of the region (as illustrated in Figure 1). Let $a_{i}$ denote the number of divided sub-regions in region ${ }_{i, j}$, then the procedure of division is implemented.

$$
a_{i}=a_{i-1}+4, \forall i=2 \ldots K \text {, where } a_{1}=4 \text {. }
$$

Specifically, the procedure divided the circular sensing area into 4, 8, 12 regions in level $L_{1}, L_{2}, L_{3}$, respectively. As for the area of region ${ }_{i, j}$, it can be derived as follows.

$$
\text { region }_{i, j}=\left\{\begin{array}{l}
\frac{\pi \times\left(\frac{i}{K} \times R\right)^{2}}{4 \times i}, \text { if } i=1 \\
\frac{\pi \times\left(\frac{i}{K} \times R\right)^{2}-\pi \times\left[\frac{(i-1)}{K} \times R\right]^{2}}{4 \times i}, \text { if } i \geq 2 .
\end{array}\right.
$$

, where $K$ is total number of levels.

In the case of $R=100 m$ and $K=3$, the area of region ${ }_{i, j}$ can be calculated as follows.

$$
\begin{aligned}
& \text { region }_{1, j}=\frac{\pi \times\left(\frac{1}{3} \times 100\right)^{2}}{4 \times 1}=\frac{10000}{36} \pi, \forall j=1 \ldots 4 . \\
& \text { region }_{2, j}=\frac{\pi \times\left(\frac{2}{3} \times 100\right)^{2}-\pi \times\left(\frac{1}{3} \times 100\right)^{2}}{4 \times 2}=\frac{30000}{72} \pi, \forall j=1 \ldots 8 . \\
& \text { region }_{3, j}=\frac{\pi \times\left(\frac{3}{3} \times 100\right)^{2}-\pi \times\left(\frac{2}{3} \times 100\right)^{2}}{4 \times 3}=\frac{50000}{108} \pi, \forall j=1 \ldots 12 \text {. (3 }
\end{aligned}
$$

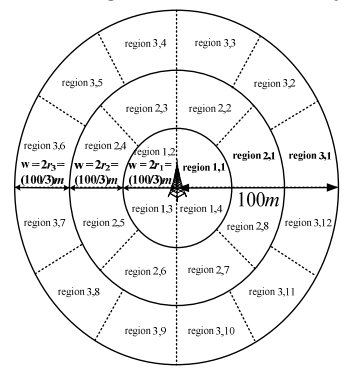

Figure 1. An example of 3 levels in the concentric model

Theorem 1: For two arbitrary regions region $_{x, j}$ and region $_{\mathrm{y}, \mathrm{j}}$, the area of region $_{\mathrm{x}, \mathrm{j}}$ is greater than the area of region $_{\mathrm{y}, \mathrm{j}}$ if $\mathrm{x}>\mathrm{y}, \forall x, y$.

The proof is given in Appendix.

Definition 1: Let N\#CTN and M, respectively, denote the number of covered tested nodes in sensing area and the total number of scattered tested nodes (scattered randomly). The coverage ratio $(\mathrm{CR})$ of the system is defined as $C R=\frac{N \# C T N}{M}$.

Consider a test node $\mathrm{TN}_{\mathrm{m}}$ and a sensor node $\mathrm{S}$, which are located at $(\mathrm{a}, \mathrm{b})$ and $(\mathrm{c}, \mathrm{d})$, respectively. The covering range of a sensor node is $R_{s}$. The possibility that a test node $T_{m}$ can be covered by a sensor node $\mathrm{S}$ can be described as:

$$
P\left(T N_{m}\right)=\left\{\begin{array}{l}
1, \text { if } \sqrt{(c-a)^{2}+(d-b)^{2}} \leq R_{s} . \\
0, \text { otherwise }
\end{array}\right.
$$

Consider a WSN with $\mathrm{N}$ sensor nodes. The probability that a test node $\mathrm{TN}_{\mathrm{m}}$ is covered is written as:

$$
C T N_{m}=1-\prod_{i=1}^{N}\left(1-P\left(T N_{m}\right)\right) \cdot
$$

Then, coverage ratio (CR) of the system is defined as:

$$
C R=\frac{\sum_{m=1}^{M} C T N_{m}}{M}=\frac{N \# C T N}{M} \text {. }
$$

More specifically, if total number of scattered tested nodes are 1000 (i.e., $M=1000$ ), and the number of covered 
tested nodes in sensing area are only 800 (i.e., N\#CTN = 800 ). Therefore, the coverage ratio is 0.8 (i.e., $\mathrm{CR}=80 \%$ ).

We assume that each region elects a sensor node to serve as the cluster head $(\mathrm{CH})$, with its location closest to the most central location. Each sensor node is assumed to be generated and transmitted one unit of data per round to the sink via $\mathrm{CH}$ by utilizing multi-hop communications.

We use the energy consumption model (Heinzelman et al. [21]). The consumed energy for transmitting $\mathrm{m}$ data unit over a distance $\mathrm{d}$ is $\mathrm{m} \times\left(\mathrm{E}_{\text {elec }}+\mathrm{E}_{\mathrm{amp}} \times \mathrm{d}^{\alpha}\right)$, where $\mathrm{E}_{\text {elec }}$ is the energy consumed in a sensor node for transmitting 1 bit of sensed data, $E_{a m p}$ is the amplifier energy (multi-path model), $\mathrm{d}$ indicates the transmission distance and $\alpha$ denotes the path loss exponent (i.e., $\alpha=4$ ).

\section{ANALYSIS OF ENERGY CONSUMPTION}

In the concentric model, each $\mathrm{CH}$ in outermost region r $_{k j}$ only copes with the sensed data from its member nodes (i.e., region $_{k, j} \times d_{k}$, where $d_{k}$ is the node density in level $k$ ). However, each $\mathrm{CH}$ in region $_{k-1, j}$ not only processes the data transmitted by its own member nodes, but they also relay data from region $_{k, j}$ (i.e., region $_{k-1, j} \times d_{k-1}+$ region $_{k, j} \times d_{k}$ $\times \frac{a_{k}}{a_{k-1}}$, where $a_{k}$ be the number of sub-regions in region $n_{k, j}$ ). The transmission distance is measured between the center of two adjacent regions for the sake of simplicity in calculations (i.e., $\left(r_{k}+r_{k-1}\right)$ in level $L_{k},\left(r_{k-1}+r_{k-2}\right)$ in level $L_{k-1}$ and so on). In addition, the distance between each $\mathrm{CH}$ (in innermost level $L_{1}$ ) and sink is only $r_{1}$. In this way, the total energy consumption of a $\mathrm{CH}$ in level $\mathrm{L}_{i}, \forall i=1 \ldots K$, can be evaluated as follows.

$$
\begin{aligned}
& E_{k}=\left[\text { region }_{k, j} \times d_{k}\right] \times\left[\mathrm{E}_{\text {elec }}+\mathrm{E}_{\text {amp }} \times\left(r_{k}+r_{k-1}\right)^{\alpha}\right] \\
& E_{k-1}=\left[\text { region }_{k-1, j} \times d_{k-1}+\text { region }_{k, j} \times d_{k} \times \frac{a_{k}}{a_{k-1}}\right] \times\left[\mathrm{E}_{\text {elec }}+\mathrm{E}_{\text {amp }} \times\left(r_{k-1}+r_{k-2}\right)^{\alpha}\right] \\
& E_{k-2}=\left[\text { region }_{k-2, j} \times d_{k-2}+\text { region }_{k-1, j} \times d_{k-1} \times \frac{a_{k-1}}{a_{k-2}}+\text { region }_{k, j} \times d_{k} \times \frac{a_{k}}{a_{k-2}}\right] \times \\
& \vdots \\
& \quad\left[\mathrm{E}_{\text {elec }}+\mathrm{E}_{\text {amp }} \times\left(r_{k-2}+r_{k-3}\right)^{\alpha}\right] \\
& E_{1}=\left[\text { region }_{1, j}+\sum_{i=2}^{k} \text { region }_{i, j} \times d_{i} \times \frac{a_{i}}{a_{1}}\right] \times\left[\mathrm{E}_{\text {elec }}+\mathrm{E}_{\text {amp }} \times\left(r_{1}\right)^{\alpha}\right]
\end{aligned}
$$

\section{PRoposed Scheme}

The proposed Rich scheme consists of three phases: node deployment phase, region formation phase and data routing phase.

\section{A. Node deployment phase}

Node deployment is one of the critical issues in WSNs. By utilizing the proposed scheme, sensor nodes can be deployed efficiently in a predetermined sensing area to achieve full coverage. The node deployment phase consists of determining the number of levels in topology and calculating the node density in each region.

- Determining the number of levels $(K)$ in topology

In the concentric model, sensing area is divided into $K$ levels. The width of each region $_{i, j}$ is set to $w$. Therefore, $\frac{R}{K}=w=2 \times r_{i}, \forall i=1 \ldots K$ (note that this parameter $(K)$ is adjustable in the Rich scheme).

\section{Calculating the node density in each region}

Deterministic deployment is used because the location of each sensor node can be predetermined appropriately. We assume that sensor nodes are deployed in each region ${ }_{i, j}$ according to the following conditions and constraints.

$$
\begin{aligned}
& \frac{\left[\text { region }_{1, j} \times d_{1}\right] \times \varepsilon_{1}}{E_{1}} \approx \frac{\left[\text { region }_{2, j} \times d_{2}\right] \times \varepsilon_{2}}{E_{2}} \approx \cdots \\
& \approx \frac{\left[\text { region }_{k-1, j} \times d_{k-1}\right] \times \varepsilon_{k-1}}{E_{k-1}} \approx \frac{\left[\text { region }_{k, j} \times d_{k}\right] \times \varepsilon_{k}}{E_{k}} \\
& \sum_{i=1}^{k}(4 \times i) \times\left[\text { region }_{i, j} \times d_{i}\right]=N_{A L L}
\end{aligned}
$$$$
\text { subject to: }\left(\text { region }_{i, j} \times d_{i}\right) \geq 1, \forall i=1 \cdots K,
$$

, where $\varepsilon_{i}$ is the initial energy of each sensor node in level $L_{i}$ (i.e., $\varepsilon_{1}=\varepsilon_{2}=\ldots=\varepsilon_{k-1}=1$ joule and an adjustable $\varepsilon_{k}$ ), $E_{i}$ is the total energy consumption of each $\mathrm{CH}$ in level $L_{i}$ (as mentioned above in Section IV) and $N_{\mathrm{ALL}}$ is the total number of sensor nodes. From (8a), we can calculate the ratio of $d_{1}$, $d_{2}, \ldots, d_{k}$. We then find out how many sensor nodes should be deployed into each region from (8a) and (8b). In order to sufficiently monitor the entire sensing area, each region must be completely covered by at least one sensor node. The coverage requirement can be guaranteed from Constraint (8c). In fact, node deployment strategies play a critical role in WSNs. The effectiveness of node deployment is to deploy the least number of sensor nodes that satisfy specific coverage requirement, or enhance the sensing field.

\section{- A numerical example}

Consider a numerical example of 3-level concentric model with $R=100 \mathrm{~m}, \varepsilon_{1}=\varepsilon_{2}=1$ joule and an adjustable $\varepsilon_{3}$ (i.e., 0.7). 300 sensor nodes are deployed in the sensing field. According to (9a), we can get the ratio of $d_{1}, d_{2}$ and $d_{3}$. The got ratio can be put in $(9 \mathrm{~b})$ to calculate the number of sensor nodes in each region in order to achieve nearly balanced energy consumption.

$$
\begin{gathered}
\frac{\left[\frac{10000}{36} \times \pi \times d_{1}\right] \times 1}{E_{1}} \approx \frac{\left[\frac{30000}{72} \times \pi \times d_{2}\right] \times 1}{E_{2}} \approx \frac{\left[\frac{50000}{108} \times \pi \times d_{3}\right] \times 0.7}{E_{3}} \\
+(4 \times 1) \times\left[\frac{10000}{36} \times \pi \times d_{1}\right]+(4 \times 2) \times\left[\frac{30000}{72} \times \pi \times d_{2}\right] \\
+(4 \times 3) \times\left[\frac{50000}{108} \times \pi \times d_{3}\right]=300
\end{gathered}
$$

subject to: $\left(\right.$ region $\left._{i, j} \times d_{i}\right) \geq 1, \forall i=1 \cdots 3$.

From the above equations, we obtain $d_{1} \approx 0.0163071$, $d_{2} \approx 0.00288291$ and $d_{3} \approx 0.00074132$. Accordingly, $\mathrm{R}_{1 \text {-total }}=$ $171, \mathrm{R}_{2 \text {-total }}=90$ and $\mathrm{R}_{3 \text {-total }}=39$, where $\mathrm{R}_{i \text {-total }}$ denotes the total number of sensor nodes in all region ${ }_{i, j}$. This example can be extended easily in any $K$-level concentric model.

\section{B. Region formation phase}

The region formation phase must ensure little overhead in terms of energy cost. Region formation phase consists of region-based intelligent $\mathrm{CH}$ selection and region setup, and $\mathrm{CH}$ rotation within the region.

- Region-based intelligent $\mathrm{CH}$ selection and region setup:

At first, each sensor node reports its current location information and its residual energy to the sink. The sink selects the ideal location (the most central location) in each region $_{i, j}$ serves as a $\mathrm{CH}$, as shown in Figure 2. The selected $\mathrm{CHs}$ broadcast the cluster head message to member nodes 
(Head_Msg). If a sensor node receives multiple Head_Msgs, a sensor node only joins the closest $\mathrm{CH}$. For those sensor nodes that do not serve as $\mathrm{CHs}$ and do not receive any Head_Msg, they may send a find message (Find_Msg) to look for the closest region to join them.

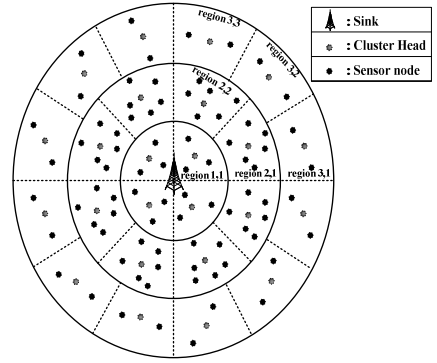

Figure 2. An illustration of the initial $\mathrm{CH}$ selected by the sink in each region

- $\mathrm{CH}$ rotation within the region:

In order not to use up the energy of initial $\mathrm{CHs}$ earily, $\mathrm{CH}$ rotation within each region is needed. The threshold of $\mathrm{CH}$ energy $\left(T_{e}\right)$ is defined. If the residual energy of the $\mathrm{CH}$ in any region $_{i, j}$ is under $T H_{e}$, the sink reselects a sensor node (with most residual energy) to serve as the new $\mathrm{CH}$. Meanwhile, the new $\mathrm{CH}$ is broadcast a change message (Change_Msg) to notice its member nodes.

\section{Data routing phase}

The data routing phase consists of intra-region data routing and inter-region data routing.

\section{- Intra-region data routing}

Consider a WSN with high density of sensor nodes, if sensor nodes employ multi-hop routing in intra-region, the transmitted data may cause lots of delay before reaching the targeted CHs. For this reason, intra-region data routing adopts direct transmissions. This can be avoided timeconsuming routing between sensor node and $\mathrm{CH}$ in order to enable time-sensitive applications of WSNs.

\section{- Inter-region data routing}

It is no doubt that the energy consumption in a radio transmission is proportional to the $\mathrm{n}$-th power of transmission distance (i.e., $\mathrm{n}=4$ ). If the $\mathrm{CH}$ transmits data to the next adjacent $\mathrm{CHs}$ utilizes direct transmissions, the $\mathrm{CH}$ may consume a lot of energy. Hence, intra-region data routing employs multi-hop transmissions. Let us specify how to obtain the relay traffic load of intra-region data routing with the goal of balancing energy among CHs.

From (1), we know that the relay traffic $\left(R T_{i-1}\right)$ of the $\mathrm{CH}$ in level $L_{i-1}$ from CHs in level $L_{i}$ is:

$$
R T_{i-1}=\frac{a_{i}}{a_{i-1}} \times D S_{i}, \forall i=2 \ldots K
$$

, where $D S_{i}$ is the data size in region $_{i, j}$.

If the calculated value $R T_{i-1}$ is an integer, the relay traffic delivered from $\mathrm{CHs}$ in level $L_{i}$ is equivalently allocated by CHs in level $L_{i-1}$. For example, 8 regions are in level $L_{2}$ (i.e., $a_{2}=8$ ) and 4 regions are in level $L_{1}$ (i.e., $a_{1}=4$ ). Therefore, each $\mathrm{CH}$ in level $L_{1}$ has relay traffic of $\frac{8}{4} \times D S_{2}$ from $\mathrm{CHs}$ in level $L_{2}$. However, if the $R T_{i-1}$ is not an integer (i.e., $a_{2}=8$ and $a_{3}=12$, then $R T_{2}=\frac{12}{8} \times D S_{3}$ ), the relay traffic of $\mathrm{CHs}$ may not be shared equivalently. In view of this, an adjustment is required to even the shares of each region. The adjustment (circular shift relaying loads in each round) is implemented as follows.

Each region in level $L_{i}$ has an equal relay traffic of $(i+1)$ $\times D S_{i+1}$ (from level $L_{i+1}$ ) in each $i$ rounds, $\forall i=1 \ldots K$.

As shown in Figure 3a, Figure $3 \mathrm{~b}$ and Table I, each region in level $L_{2}$ has equal relay traffic of $3 \times D S_{3}$ (from level $L_{3}$ ) in each 2 rounds. In this way, the relay traffic can be allocated equally.

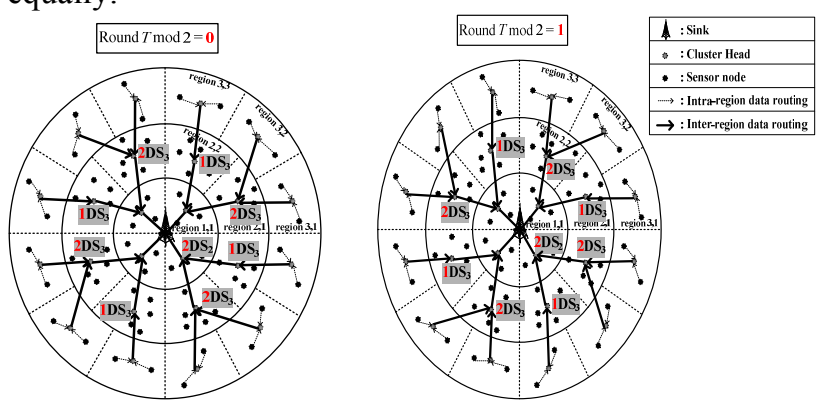

Figure 3a\&3b. Examples of relay traffic from $L_{3}$ to $L_{2}$ in each 2 rounds

TABLE I. AN EXAMPLE OF RELAY TRAFFIC FROM LEVEL $L_{3}$ TO $L_{2}$ IN EACH 2 ROUNDS

\begin{tabular}{|c|c|c|c|c|}
\hline$L_{3} \rightarrow L_{2}$ & region:a & region:2: & region $_{3}$ & region:s \\
\hline Round $T \bmod 2=0$ & $2 \mathrm{DS}_{3}$ & $1 \mathrm{DS}_{3}$ & $2 \mathrm{DS}_{3}$ & $1 \mathrm{DS}_{3}$ \\
\hline $\operatorname{Round} T \bmod 2=1$ & $\mathrm{DSS}_{3}$ & $\mathrm{DSS}_{3}$ & $x_{1 D S_{3}}$ & $\mathrm{ADS}_{3}$ \\
\hline 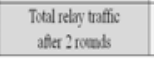 & $3 \mathrm{DS}_{3}$ & $3 \mathrm{DS}_{3}$ & $3 \mathrm{DS}_{3}$ & $3 \mathrm{DS}_{\mathrm{s}}$ \\
\hline
\end{tabular}

\section{Performance Evaluation}

In this section, we evaluate the proposed Rich scheme, EBDA scheme (Senouci et al., 2011), and Random scheme using MATLAB. Note that Random scheme utilizes the same region formation phase and data routing phase as compared to Rich scheme, except for the sensor nodes deployment strategy (i.e., Random scheme scatters sensor node randomly). We are interested in the following metrics:

(1) coverage ratio;

(2) network lifetime and

(3) the number of sensor nodes still alive over rounds.

Coverage ratio is described in Definition 1. 'Network lifetime' is defined as the elapsing time until first sensor node exhaust its energy, and is measured in 'rounds'. The definition of a 'round' is that the sensor node transmits the sensed data via the $\mathrm{CHs}$ to the sink. All the parameters are given as follows. $E_{\text {elec }}=50 \mathrm{~nJ} / \mathrm{bit}, \varepsilon_{\text {amp }}=0.0013 \mathrm{pJ} / \mathrm{bit} / \mathrm{m}^{4}$, the path loss exponent is 4 ; total radius in the concentric model is 100 meters (i.e., $R=100 \mathrm{~m}$ ); initial energy of each sensor node is 1 joule, except for the sensor nodes in outermost regions (i.e., $\varepsilon_{1}=\varepsilon_{2}=1$ and $\varepsilon_{3}=0.7$ joule); each sensor node transmits 800 bits of data per round to the sink via multi-hop communications. We averaged the results of 1000 runs for each scenario.

\section{A. Coverage ratio}

The simulation is performed to evaluate the coverage ratio with the varying number of sensor nodes. In scenario 1 , total number of scattered tested nodes are 100 (i.e., $M=$ $100)$, and covered range of each sensor node is 10 meters (i.e., $R_{s}=10 \mathrm{~m}$ ); in scenario 2 , total number of scattered tested nodes are 200 (i.e., $M=200$ ), and covered range of each sensor node is 20 meters (i.e., $R_{s}=20 \mathrm{~m}$ ).

Figure 4 shows the simulation result for scenario 1 in comparison with the proposed Rich, EBDA scheme, and Random scheme when the number of sensor nodes are 
increased from 100 to 300 . It can be observed that the coverage ratio of the Rich is over $96 \%$. The reason for this result is due to deployment manner (as mentioned in $8 \mathrm{a}-8 \mathrm{c}$ ). Furthermore, coverage ratio has an increasing trend as the sensor nodes increases. This is because when the sensor nodes increase, the sensing range of the system is increased. Therefore, coverage ratio is increased.

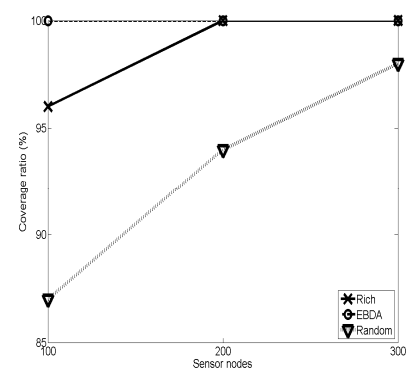

Figure 4. Coverage ratio for scenario 1 comparison between the Rich scheme, EBDA scheme and Random scheme

We can see that the coverage ratio of the EBDA scheme is highest. To achieve the full coverage ratio (i.e., 100\%), the EBDA scheme only requires 100 sensor nodes, whereas the proposed Rich scheme requires more than 100 sensor nodes. As for the Random scheme, the node deployment strategy is to scatter sensor nodes randomly in the sensing area. Thus, coverage ratio is lowest.

Next, we consider the case in scenario 2, as depicted in Figure 5. In all cases, the EBDA scheme needs fewer sensor nodes than Rich scheme and Random scheme to obtain full coverage ratio. Furthermore, it can be observed that Rich scheme and Random scheme have a slightly larger coverage ratio compared to scenario 1 . The reason for this is that scenario 2 sets a larger covered range of each sensor node.

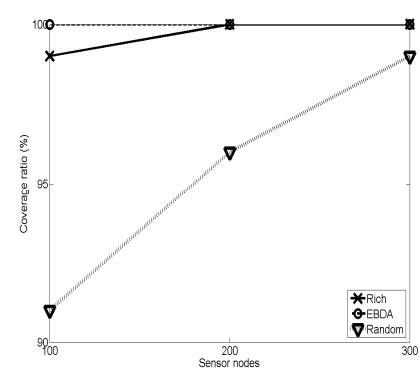

Figure 5. Coverage ratio for scenario 2 comparison between the Rich scheme, EBDA scheme and Random scheme

TABLE II. NETWORK LIFETIME COMPARISON BETWEEN THE RICH SCHEME, EBDA SCHEME AND RANDOM SCHEME

\begin{tabular}{|l|c|c|c|}
\hline \multicolumn{1}{|c|}{ Sensor nodes } & $\mathbf{1 0 0}$ & $\mathbf{2 0 0}$ & $\mathbf{3 0 0}$ \\
\hline Rich & & & \\
\hline EBDA & 3276 & 3043 & 2894 \\
\hline Random & 2642 & 2395 & 2203 \\
\hline
\end{tabular}

\section{B. Network lifetime}

Table II demonstrates that the network lifetime of Rich is longer than that of the other schemes. In Rich, the first node use up its energy occurs after 3276 rounds, 3043 rounds and 2894 rounds when the total number of sensor nodes $\left(N_{\mathrm{ALL}}\right)$ is set to 100,200 and 300 , respectively. When the system deployed 100 sensor nodes, Rich extends the network lifetime about 24\% compared to the EBDA scheme, and 96\% compared to the Random scheme. In fact, the same trends are for $N_{\mathrm{ALL}}$ is 300 . This is due to the fact that Rich appropriately deploys sensor nodes in each region according to the energy consumption of each $\mathrm{CH}$. Furthermore, most of sensor nodes consume the energy evenly in the Rich scheme. Hence, balancing energy dissipation of the networks is achieved.

\section{The number of sensor nodes still alive}

Figure 6 shows the number of sensor nodes still alive over rounds. The number of nodes still alive in Rich scheme is greater than that of the other protocols. This is because Rich scheme computes the ideal node density for each region to balance the energy consumption of each $\mathrm{CH}$. Therefore, the first sensor node uses up its energy later than in both EBDA scheme and Random scheme. These results verify the results appeared in Table II.

Moreover, the slope of the curve in Rich scheme is steep than in the EBDA scheme and Random scheme; this phenomenon explains that Rich scheme utilizes circular shift algorithm (as stated in Section V.C) to allocate relay traffic equally.

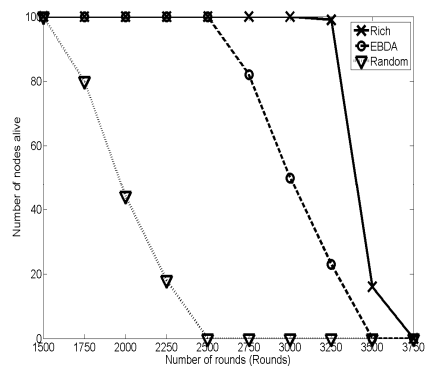

Figure 6 . The number of sensor nodes still alive over rounds

\section{CONCLUSION}

In concentric-based multi-hop WSNs, CHs closer to the sink have larger relay traffic than those $\mathrm{CHs}$ farther away from the sink. In order to avoid unbalanced traffic pattern in WSNs, this paper proposes "Region-based Intelligent Cluster-Head selection and node deployment strategy in concentric-based WSNs (called Rich)". According to the relay traffic of each $\mathrm{CH}$, we can find out how many sensor nodes should be deployed in each region so as to each region can exhaust its energy at approximately the same time. This paper further proposes circular shift routing in inter-region, which further leads to balanced energy consumption among CHs. Moreover, the coverage ratio, energy consumption issues are taken into consideration in the proposed Rich scheme. Simulation results demonstrates that the proposed Rich scheme mitigates the 'energy hole problem' significantly, extends the network lifetime in comparison with the EBDA scheme and Random scheme.

\section{APPENDIX A}

This appendix shows that the area of region r $_{x, j}$ is greater than the area of region , if $_{j}>y, \forall 1<x, y<k$. We prove the theorem via mathematical induction.

Theorem 1: For two arbitrary regions region rej $_{x, j}$ and region $_{y, j}$, the area of region $x_{x, j}$ is greater than the area of region $_{y, j}$ if $x>y, \forall x, y$.

Proof: According to (2), 


$$
\begin{aligned}
& \text { region }_{x, j}=\frac{\pi \times\left(\frac{x}{K} \times R\right)^{2}-\pi \times\left[\frac{(x-1)}{K} \times R\right]^{2}}{4 \times x} \text { and } \\
& \text { region }_{y, j}=\frac{\pi \times\left(\frac{y}{K} \times R\right)^{2}-\pi \times\left[\frac{(y-1)}{K} \times R\right]^{2}}{4 \times y}
\end{aligned}
$$

Let $(\mathrm{x}-\mathrm{y})$ be the $N, \forall N \in$ integer.

Base case:

Show that region $_{x, j}>$ region $_{y, j}$ when $N=1$ (i.e., $\mathrm{x}-\mathrm{y}=\mathbf{1}$ ).

Without loss of generality, we assume that $\mathrm{x}=\mathrm{y}+1$.

region $_{x, j}=$ region $_{y+1, j}=\frac{\pi \times\left(\frac{y+1}{K} \times R\right)^{2}-\pi \times\left\{\frac{[(y+1)-1]}{K} \times R\right\}^{2}}{4 \times(y+1)}=\frac{\pi \times\left[\left(\frac{2 y+1}{K} \times R\right) \times\left(\frac{R}{K}\right)\right]}{4 \times(y+1)}$.

$$
\frac{\text { region }_{x, j}}{\text { region }_{y, j}}=\frac{\left\{\frac{\pi \times\left[\left(\frac{2 y+1}{K} \times R\right) \times\left(\frac{R}{K}\right)\right]}{4 \times(y+1)}\right\}}{\left\{\frac{\pi \times\left(\frac{y}{K} \times R\right)^{2}-\pi \times\left[\frac{(y-1)}{K} \times R\right]^{2}}{4 \times y}\right\}}=\frac{2 y^{2}+y}{2 y^{2}+y-1}>1 .
$$

Hence, region $_{x, j}>$ region $_{y, j}$ when $\mathrm{x}-\mathrm{y}=1$.

\section{Base case:}

Show that region $_{x, j}>$ region $_{y, j}$ when $\boldsymbol{N}=2$ (i.e., $\mathrm{x}-\mathrm{y}=2$ ).

Without loss of generality, we assume that $\mathrm{x}=\mathrm{y}+2$.

$$
\begin{aligned}
& \text { region }_{x, j}=\text { region }_{y+2, j}=\frac{\pi \times\left(\frac{y+2}{K} \times R\right)^{2}-\pi \times\left\{\frac{[(y+2)-1]}{K} \times R\right\}^{2}}{4 \times(y+2)}=\frac{\pi \times\left[\left(\frac{2 y+3}{K} \times R\right) \times\left(\frac{R}{K}\right)\right]}{4 \times(y+2)} \text {. }
\end{aligned}
$$



Hence, region $_{x, j}>$ region $_{y, j}$ when $\mathrm{x}-\mathrm{y}=2$.

Inductive hypothesis:

Assume that region $_{x, j}>$ region $_{y, j}$ for $\boldsymbol{N}=\mathbf{n}$ (i.e., $\mathrm{x}-\mathrm{y}=\mathbf{n}$ ).

Without loss of generality, we assume that $x=y+n$. That

is, in this case, we assume that $\frac{\text { region }_{x, j}}{\text { region }_{y, j}}>1$ when $\mathrm{x}=\mathrm{y}+\mathrm{n}$.

Inductive step:

Show that region $_{x, j}>$ region $_{y, j}$ for $\boldsymbol{N}=\mathbf{n}+\mathbf{1}$ (i.e., $\mathrm{x}-\mathrm{y}=\mathbf{n}+\mathbf{1}$ ).

Without loss of generality, we assume that $\mathrm{x}=\mathrm{y}+\mathrm{n}+1$.

$$
\begin{aligned}
& \text { region }_{x, j}= \text { region }_{y+n+1, j}=\frac{\pi \times\left(\frac{y+n+1}{K} \times R\right)^{2}-\pi \times\left\{\frac{[(y+n+1)-1]}{K} \times R\right\}^{2}}{4 \times(y+n+1)} \\
&= \frac{\pi \times\left[\left(\frac{2 y+2 n+1}{K} \times R\right) \times\left(\frac{R}{K}\right)\right]}{4 \times(y+n+1)} . \\
& \frac{\text { region }}{x, j}_{\text {region }_{y, j}}=\frac{\left\{\frac{\pi \times\left[\left(\frac{2 y+2 n+1}{K} \times R\right) \times\left(\frac{R}{K}\right)\right]}{4 \times(y+n+1)}\right\}}{\pi \times\left(\frac{y}{K} \times R\right)^{2}-\pi \times\left[\frac{(y-1)}{K} \times R\right]^{2}}-\frac{2 y^{2}+y}{2 y^{2}+y-(n+1)}>1 .
\end{aligned}
$$

Consequently, the proof of this theorem is completed.

\section{REFERENCES}

[1] C. W. Chen and Y. Wang, "Chain-Type Wireless Sensor Network for Monitoring Long Range Infrastructures: Architecture and Protocols," Int. J. of Distrib. Sensor Networks, vol. 4, no. 4, pp. 287-314, Oct. 2008. doi: http://dx.doi.org/10.1080/15501320701260261

[2] W. Fang, S. Li, X. Liang and Z. Li, "Cluster-based Data Gathering in Long-Strip Wireless Sensor Networks," Advances in Electrical and Computer Engineering, vol. 12, no. 1, pp. 3-8, Feb. 2012. doi: http://dx.doi.org/10.4316/AECE.2012.01001

[3] M. Saleem, I. Ullah and M. Farooq, "BeeSensor: An energy-efficient and scalable routing protocol for wireless sensor networks," Information Sciences, vol. 200, no. 1, pp. 38-56, Jan. 2012. doi: http://dx.doi.org/10.1016/j.ins.2012.02.024

[4] M.S. Familiar, J.F. Martinez, I. Corredor and C.G.-Rubio, "Building service-oriented Smart Infrastructures over Wireless Ad Hoc Sensor Networks: A middleware perspective," Computer Networks, vol. 56, no. 4, pp.1303-1328, Mar. 2012. doi: http://dx.doi.org/10.1016/j.comnet.2011.12.005
[5] D. Tian, Z. Lei and N.D. Georganas, "Configuring node status in a two-phase tightly integrated mode for wireless sensor networks," Int. J. of Ad Hoc and Ubiquitous Computing, vol. 2, no.3, pp. 175-185, 2007. doi: http://dx.doi.org/10.1504/IJAHUC.2007.012436

[6] N. Essaddi, M. Hamdi, S. Habib and N. Boudriga, "Evolutionary strategies for non-uniform deployment in wireless sensor networks," Int. J. of Communication Networks and Distrib. Systems, vol. 7, no. 3/4, pp. 331-354, May 2011. doi http://dx.doi.org/10.1504/IJCNDS.2011.042383

[7] A. Gorbenko, M. Mornev, V. Popov and A. Sheka, "The problem of sensor placement for triangulation-based localisation," Int. J. of Automation and Control, vol. 5, no. 3, pp. 245-253, Oct. 2011. doi: http://dx.doi.org/10.1504/IJAAC.2011.042855

[8] G. Fan, R. Wang, H. Huang, L. Sun and C. Sha, "CoverageGuaranteed Sensor Node Deployment Strategies for Wireless Sensor Networks," Sensors, vol. 10, no. 3, pp. 2064-2087, Mar. 2010. doi: http://dx.doi.org/doi:10.3390/s100302064

[9] S. C. Liu, "A Lifetime-Extending Deployment Strategy for Multi-Hop Wireless Sensor Networks," Proceedings of the 4th Annual Communication Networks and Services Research Conf. (CNSR), pp. 53-60, May 2006. doi: http://dx.doi.org/10.1109/CNSR.2006.7

[10] M. Maleki and M. Pedram, "QoM and Lifetime-Constrained Random Deployment of Sensor Networks for Minimum Energy Consumption," The 4th Int. Symposium on Information Processing in Sensor Networks (IPSN), pp. 293-300, Apr. 2005. doi: http://dx.doi.org/10.1109/IPSN.2005.1440938

[11] Y. Xin, T. Guven and M. Shayman, "Relay Deployment and Power Control for Lifetime Elongation in Sensor Networks," Proceedings of the IEEE Int. Conf. on Communications (ICC), pp. 3461-3466, June 2006. doi: http://dx.doi.org/10.1109/ICC.2006.255608

[12] N. Kulkarni, R. Prasad, H. Cornean and N. Gupta, "Performance Evaluation of AODV, DSDV and DSR for Quasi Random Deployment of Sensor Nodes in Wireless Sensor Networks," Int Conf. on Devices and Communications (ICDeCom), pp. 1-5, Feb. 2011. doi: http://dx.doi.org/10.1109/ICDECOM.2011.5738571

[13] Y.R. Tsai, "Sensing Coverage for Randomly Distributed wireless sensor networks in shadowed environments," IEEE Trans. on Vehicular Technology, pp. 556-564, Jan. 2008. doi: http://dx.doi.org/10.1109/TVT.2007.905624

[14] P. Balister and S. Kumar, "Random vs. Deterministic Deployment of Sensors in the Presence of Failures and Placement Errors," IEEE Int. Conf. on Computer Communications (INFOCOM), pp. 2896-2900, Apr. 2009. doi: http://dx.doi.org/10.1109/INFCOM.2009.5062254

[15] G. Chen, S.K. Das and X. Wu, "Avoiding energy holes in wireless sensor networks with nonuniform node distribution," IEEE Trans. on Parallel and Distrib. Systems, vol.19, no.5, pp.710-720, May 2008 doi: http://dx.doi.org/10.1109/TPDS.2007.70770

[16] D. Wang, B. Xie and D.P. Agrawal, "Coverage and lifetime optimization of wireless sensor networks with Gaussian distribution," IEEE Trans. on Mobile Computing, vol. 7, no. 12, pp. 1444-1458, Dec. 2008. doi: http://dx.doi.org/10.1109/TMC.2008.60

[17] F.A. Aderohunmu, J.D. Deng, and M.K. Purvis, "A Deterministic Energy-efficient Clustering Protocol for Wireless Sensor Networks," Int.1 Conf. on Intelligent Sensors, Sensor Networks and Information Processing (ISSNIP), pp. 341-346, Dec. 2011. doi: http://dx.doi.org/10.1109/ISSNIP.2011.6146592

[18] D. Ganesan, R. Cristescu and B.B. Lozano, "Power Efficient Sensor Placement and Transmission Structure for Data Gathering under Distortion Constraints," ACM Trans. on Sensor Networks, vol. 2, no. 2, pp. 155-181, May $2006 . \quad$ doi: http://dx.doi.org/10.1145/1149283.1149284

[19] W. K. Lai, C. S. Fan and L. Y. Lin, "Arranging cluster sizes and transmission ranges for wireless sensor networks," Information Sciences, vol. 183, no. 1, pp. 117-131, Jan. 2012. doi: http://dx.doi.org/10.1016/j.ins.2011.08.029

[20] M.R. Senouci, A. Mellouk, L. Oukhellou and A. Aissani, "Uncertainty-Aware Sensor Network Deployment," IEEE Global Telecommunications Conf. (GLOBECOM), pp. 1-5, Dec. 2011. doi http://dx.doi.org/10.1109/GLOCOM.2011.6134363

[21] W.R. Heinzelman, A. Chandrakasan and H. Balakrishnan, "An application-specific protocol architecture for wireless microsensor networks," IEEE Trans. on Wireless Communications, vol. 1, no. 4 pp. 660-670, Oct. 2002. doi http://dx.doi.org/10.1109/TWC.2002.804190 